\title{
Design of Cascaded Substrate Integrated waveguide Filter
}

\author{
Soumya Jain, Agya Mishra
}

\begin{abstract}
In this work a new approach to design a substrate integrated waveguide filter is presented to control the propagation constant of the filter by cascading multiple stages of substrate integrated waveguide using Iris topology. Substrate integrated waveguide have become center of attraction from past few years because of its design flexibility and compact size. First a single unit of SIW filter is designed for a set of design parameters and analyzed as consecutive rectangular waveguides. Then a single stage SIW Filter is designed by selecting suitable iris window and experimented for insertion loss for various materials as substrate from lower relative permittivity to higher, afterwards multiple stage of SIW of same length, with varying iris window is cascaded to obtain filtering function. A fitness function is defined to check the efficiency of proposed model by comparing it with rectangular waveguide structure. The proposed design is then experimented for propagation constant and insertion loss for various stages and at last the proposed design is compared with existing model on the basis of transition duration of propagation constant.
\end{abstract}

Keywords : Insertion loss, Iris Window, Propagation Constant, Relative Permittivity, Substrate Integrate Waveguide (SIW), S21 Parameter, Transition duration.

\section{INTRODUCTION}

A strong communication system is always depended on the type of transmission medium and for microwave frequency waveguides have been played a very important role form past few decades but those metallic conductor tube waveguides was so bulky they make whole communication system very voluminous an expensive to manufacture. Hence Substrate Integrate Waveguide (SIW) immerges as an alternate solution which is nothing but a compact version of these metallic conductor tube waveguide and every microwave component is been design based on this technology. Because of the inherent structural flexibility in coupling design and topological arrangement, substrate integrated waveguide (SIW) topologies shows better frequency selectivity and it is very easy to design filter using various methods, like space mapping technique [3], coupling structures [5] [7], with Defected ground structure [4], using Frequency Selective Structure [6], Perdistortion Technique [9].

Soumya Jain, Department of Electronics and telecommunication Jabalpur Engineering College ,Jabalpur, India.

Agya Mishra, department, Department of Electronics and telecommunication Jabalpur Engineering College ,Jabalpur, India
Other then these work [3]-[9], A significant effort has been devoted in past years to the development of SIW filters. Currently, the research is oriented towards the losses like return loss and insertion loss, control propagation constant is still an area of research.

In this work a new approach to design a SIW base filter has been proposed to control the propagation constant by cascading multiple stages of SIW of same length, with varying iris window to control the Propagation Constant. To calculate Propagation Constant, a single unit of SIW is approximate as equivalent rectangular waveguides [1].

\section{CONCEPT OF SUBSTRATE INTEGRATE WAVEGUIDE}

Substrate integrate waveguide is a planar transmission line with inherited properties of rectangular waveguide. The physical structure of SIW is shown in fig 1. A substrate material is sandwiched between two thin sheets of metal and instead of solid side walls an array of cylindrical metalize holes are integrate on substrate to create a guiding path. For the design proposed in this work, the parameters shown in Fig 1 play important role in designing SIW where ' $p$ ' is the period between the cylindrical holes, ' $d$ ' is diameter of cylindrical holes, ' $\mathrm{W}$ ' is center to center distance between cylindrical holes of both the rows, ' $\varepsilon_{y}$ ' relative permittivity of substrate. For analysis SIW is converted into rectangular waveguide which intern make it easy to calculate the propagation constant and losses [1] also for the design point of view the choice of suitable material as substrate is equally important.

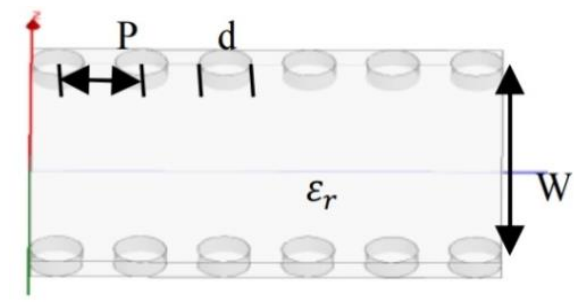

Fig.1 Structure of SIW.

\section{PROPOSED MODEL}

The flowchart of design process is shown in Fig. 2. First the SIW is represented by the equivalent rectangular waveguide model [1] then suitable Iris window is selected by hit an trial approach then single stage SIW Filter is designed and experimented for insertion loss for different materials as substrate to check if this model can be design for different substrate material, after that multiple cascaded stages SIW Filter is designed and experimented for propagation constant 


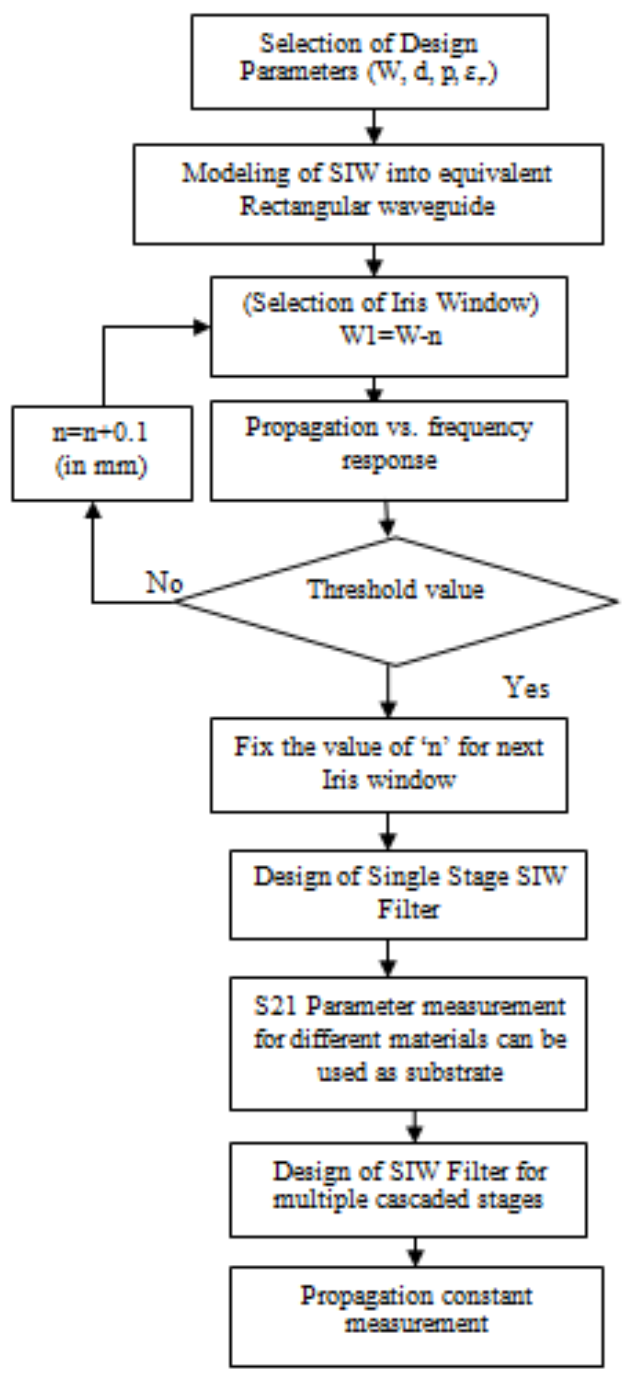

Fig. 2. Flowchart of Design of Proposed model

The design parameters for model is discussed previously. The formula se to calculate propagation constant is a function of these design parameters of SIW [1]. A period of geometrical configuration of SIW structure is represented by two cascaded rectangular waveguide, shown in Fig 3 [1].The first section consist of a rectangular waveguide of length L1 and width $\mathrm{W} 1$, the second section consist of a rectangular waveguide of length $\mathrm{L} 2$ and width $\mathrm{W} 2 . \quad \beta_{1}$ and are the propagation constant of first and second section respectively. The overall propagation constant $(\beta)$ is calculated by (4), which is a function of geometry of SIW and parameter of material used as substrate [1].

$$
\begin{aligned}
& {\left[\begin{array}{ll}
A & B \\
C & D
\end{array}\right]=\left[\begin{array}{cc}
\cos \left(\beta_{m} L_{m}\right) & \frac{j \beta_{m}}{\omega \mu} \sin \left(\beta_{m} L_{m}\right) \\
\frac{j \beta_{m}}{\omega \mu} \sin \left(\beta_{m} L_{m}\right) & \cos \left(\beta_{m} L_{m}\right)
\end{array}\right]} \\
& W_{m}=\left\{\begin{array}{cc}
(W-d) \text { for } m=1 \\
W \quad \text { for } m=2
\end{array}\right. \\
& L_{m}=\left\{\begin{array}{cc}
d \quad \text { for } m=1 \\
(s-d) \quad \text { for } m=2
\end{array}\right. \\
& \beta_{m}=\sqrt{\omega^{2} \mu \varepsilon-\left(\frac{\pi}{W_{m}}\right)^{2},} \quad \mathrm{~m}=1,2
\end{aligned}
$$

$$
\begin{aligned}
\cosh (\mathrm{j} \beta \mathrm{p})=\frac{\mathrm{A}+\mathrm{D}}{2}= & \cos \left(\beta_{1} L_{1}\right) \cos \left(\beta_{2} L_{2}\right) \\
& -\frac{1}{2}\left(\frac{\beta_{1}}{\beta_{2}}+\frac{\beta_{2}}{\beta_{1}}\right) \sin \left(\beta_{1} L_{1}\right) \sin \left(\beta_{2} L_{2}\right)
\end{aligned}
$$

ABCD matrix of one cell (Fig. 3) can be calculated by product of $A B C D$ matrices of two cascaded section. The Insertion loss (IL) in terms of $S$ parameter for the proposed model is calculated by (5).

$$
\mathrm{IL}=-20 \log |\mathrm{S} 21| \mathrm{dB}
$$

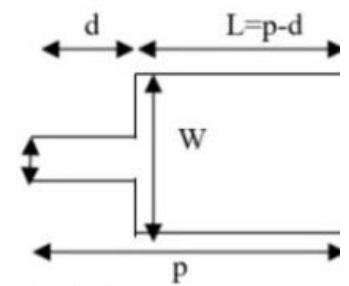

fig. 3. Equivalent rectangular waveguide representation of SIW structure.(top view) [1]
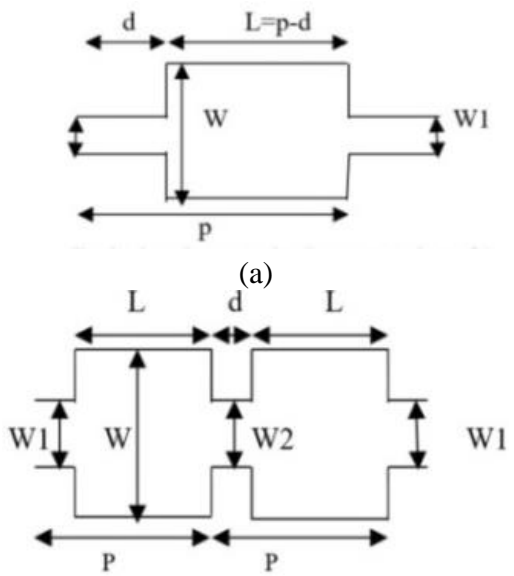

(b)

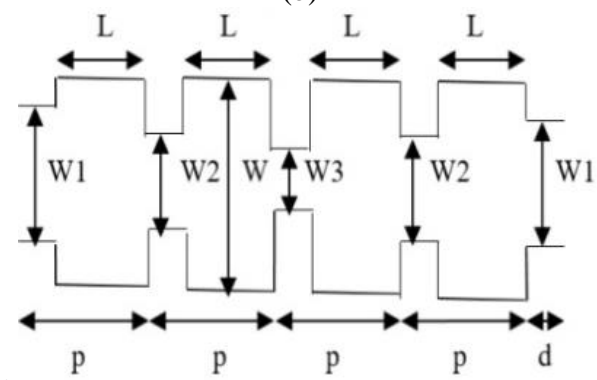

(c)

fig. 4. (a) Equivalent Rectangular Representation of Single Stage SIW Filter (b) Equivalent Rectangular Representation of SIW Filter Two cascaded stages (c) Equivalent Rectangular Representation of SIW Filter Three Cascaded stages.

For the selection of Iris window we reduce the width of SIW (W) by value ' $\mathrm{n}$ ' where $\mathrm{n}=0.1,0.2,0.3, .$. (in $\mathrm{mm}$ ) and experimented for propagation constant . By this hit and trial approach we select a suitable Iris window at which we get the threshold value and fix the value of ' $n$ ' for that window. Threshold value for the design will be the width of iris window at which it shows flat peak over certain range of frequency. Fig. 2 shows the flowchart of the process. Once a value of ' $n$ ' is selected, next Iris window is calculated by

$$
\mathrm{W} 2=\mathrm{W} 1-\mathrm{n}
$$




\section{$\mathrm{W} 3=\mathrm{W} 2-\mathrm{n}$}

Where $\mathrm{W} 1, \mathrm{~W} 2$, and $\mathrm{W} 3$ are the first, second and third Iris window, respectively. After selecting a suitable Iris window single stage SIW Filter is designed and Insertion loss is for different substrate calculated by (5). Fig. 4 shows the equivalent rectangular waveguide representation of filter at each stages.

\section{A.Efficiency Check:}

A fitness function is introduced that shows the matching percentage between proposed model (with varying Iris window) and model when all the Iris windows are of same length (that is $\mathrm{W}=\mathrm{W} 1=\mathrm{W} 2=\mathrm{W} 3$ ) that will become a rectangular waveguide. The fitness function is given by (8)

$$
\text { fitness }(\mathrm{E})=\frac{\underline{T}^{r}}{q} \times 100
$$

Where, $\mathrm{Q}^{\prime}=$ loaded $\mathrm{Q}$ factor of proposed model and $\mathrm{Q}=$ loaded $\mathrm{Q}$ factor of rectangular waveguide model. single stage SIW Filter is designed. To check the performance of propose model, single stage SIW filter is experimented for insertion loss for various substrate material, ranges lower to high relative permittivity. Then in the next step two stage cascaded SIW Filter is designed and finally three stages cascaded SIW Filter is proposed shown in fig 4, Measuring for propagation constant, represented by greek alphabet " $\beta$ " (Beta) and insertion loss.

\section{A.Selection of Iris Window :}

This step plays a crucial part in whole process as width of iris for all the stages is calculated by it. We reduce the $\mathrm{W}$ by value ' $n$ ' then calculate Beta vs Frequency response. The values of beta for various windows are given in table I. We select the window length for which propagation gives flat peak response over a range of frequency. Fig. 5 shows the propagation constant for various iris windows. As it is clear from the fig 7 , that $\mathrm{W} 1=3.07 \mathrm{~mm}$ shows comparative flat peak response between frequency ranges from $32 \mathrm{GHz}$ to $33 \mathrm{GHz}$. Hence width of first iris window is $3.07 \mathrm{~mm}$ and the difference ' $\mathrm{n}$ ' $=0.9 \mathrm{~mm}$.

\section{EXPERIMENTAL RESULTS}

The physical parameters of proposed design are $\mathrm{W}=3.97 \mathrm{~mm}, \mathrm{~d}=0.635 \mathrm{~mm}, \mathrm{p}=1.016 \mathrm{~mm}$ and $\varepsilon_{\mathrm{r}}=9.9$ [1]. First a suitable Iris window is selected as discussed previously and

Table- I Selection of Iris Window

\begin{tabular}{|c|c|c|c|c|c|c|c|c|}
\hline \multirow{2}{*}{$\begin{array}{r}\text { S } \\
\text { No }\end{array}$} & \multirow{2}{*}{$\begin{array}{c}\text { Frequency } \\
\quad \text { (GHz) }\end{array}$} & \multicolumn{7}{|c|}{ Propagation Constant (rad/m) } \\
\hline & & $\mathrm{W} 1=3.47 \mathrm{~mm}$ & $\mathrm{~W} 1=3.37 \mathrm{~mm}$ & $\mathrm{~W} 1=3.27 \mathrm{~mm}$ & $\mathrm{~W} 1=3.17 \mathrm{~mm}$ & $\mathrm{~W} 1=3.07 \mathrm{~mm}$ & $\mathrm{~W} 1=2.97 \mathrm{~mm}$ & $\mathrm{~W} 1=2.87 \mathrm{~mm}$ \\
\hline 1 & 10 & 0 & 0 & 0 & 0 & 0 & 0 & 0 \\
\hline 2 & 15 & 451.13 & 407.06 & 352.7 & 281.3 & 171.8 & 0 & 0 \\
\hline 3 & 20 & 982.07 & 962.64 & 940.9 & 316.6 & 882 & 857.9 & 822 \\
\hline 4 & 25 & 1393.9 & 1380.3 & 1365.3 & 1348.7 & 133.03 & 1307.7 & 1286.6 \\
\hline 5 & 30 & 1772 & 1761.4 & 1749.9 & 1732.2 & 1732.2 & 1707.6 & 1690.2 \\
\hline 6 & 35 & 1676.6 & 1681.6 & 1691.6 & 1702.7 & 1715 & 1728.8 & 1744.5 \\
\hline 7 & 40 & 1319.5 & 1372.2 & 1333.5 & 1344.9 & 1355.5 & 1355.5 & 1379.2 \\
\hline
\end{tabular}

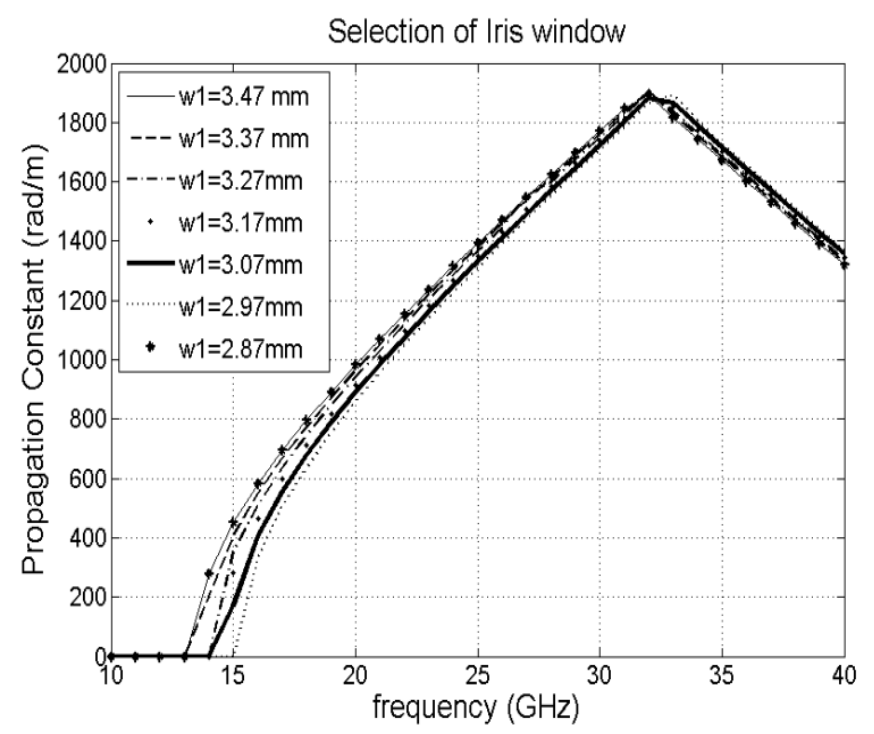

\section{B. Performance for Single stage SIW Filter:}

The geometrical values for single stage filter as shown in Fig 4 (a) are $\mathrm{W}=3.97 \mathrm{~mm}, \mathrm{~d}=0.635 \mathrm{~mm}, \mathrm{p}=1.016 \mathrm{~mm}, \mathrm{~L}=(\mathrm{p}-\mathrm{d})$ and $\mathrm{W} 1=(\mathrm{W}-\mathrm{n})=3.07 \mathrm{~mm}$. The propagation constant is calculated by (4), which is a function of geometrical parameters of SIW. Propagation constant is shown in fig. 6, which shows the maximum value of Beta is $1882.5 \mathrm{rad} / \mathrm{m}$. To check the performance of propose model, single stage SIW filter is experimented for insertion loss for various substrate material, ranges lower to high relative permittivity gives in fig 5 which shows that the proposed design is suitable for alumina $99 \%$ as it gives minimum loss early as compare to other materials between $32 \mathrm{GHz}$ to $33 \mathrm{GHz}$, rang at which we get the flat peak response, it also proves that high permittivity material greater then mica $\left(\varepsilon_{F}\right)$ can also be used as substrate for good performance.

Fig. 4 Selection of Iris window. 
S21 parameter for different material at single stage

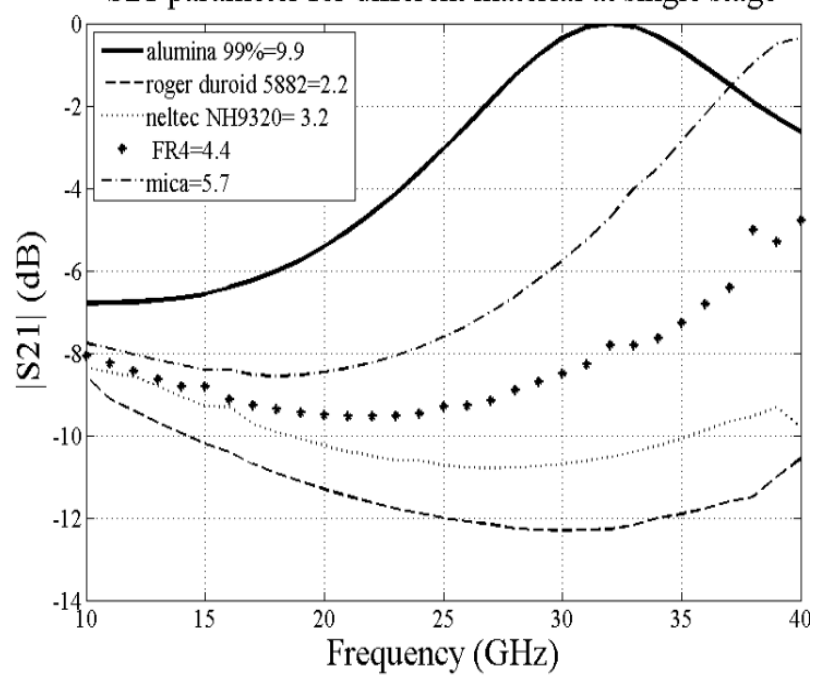

Fig. 5 Insertion loss for different materials as substrate.

\section{C.Performance for Two stage SIW filter :}

Iris window for two stage cascaded SIW filter is $\mathrm{W}=3.97 \mathrm{~mm}, \mathrm{~W} 1=3.07 \mathrm{~mm}, \mathrm{~W} 2=2.17 \mathrm{~mm}(6)$ and equivalent rectangular structure is shown in fig 4. plot for propagation constant is shown in fig 4(b). Maximum value of propagation for this design is $1036.1 \mathrm{rad} / \mathrm{m}$ which is less than maximum propagation constant for single stage.

\section{Performance for Three stage SIW filter :}

Iris window for three stage cascaded filter are $\mathrm{W}=3.97$ $\mathrm{mm}, \mathrm{W} 1=3.07 \mathrm{~mm}, \mathrm{~W} 2=2.17 \mathrm{~mm}$ and from $(7), \mathrm{W} 3=1.27 \mathrm{~mm}$. fig. 4(c) shows the equivalent structure for three stage cascaded SIW filter. fig 5 shows the plots for propagation constant, the value of maximum propagation is $668.57 \mathrm{rad} / \mathrm{m}$ for larger frequency rang as compare to other two stages.

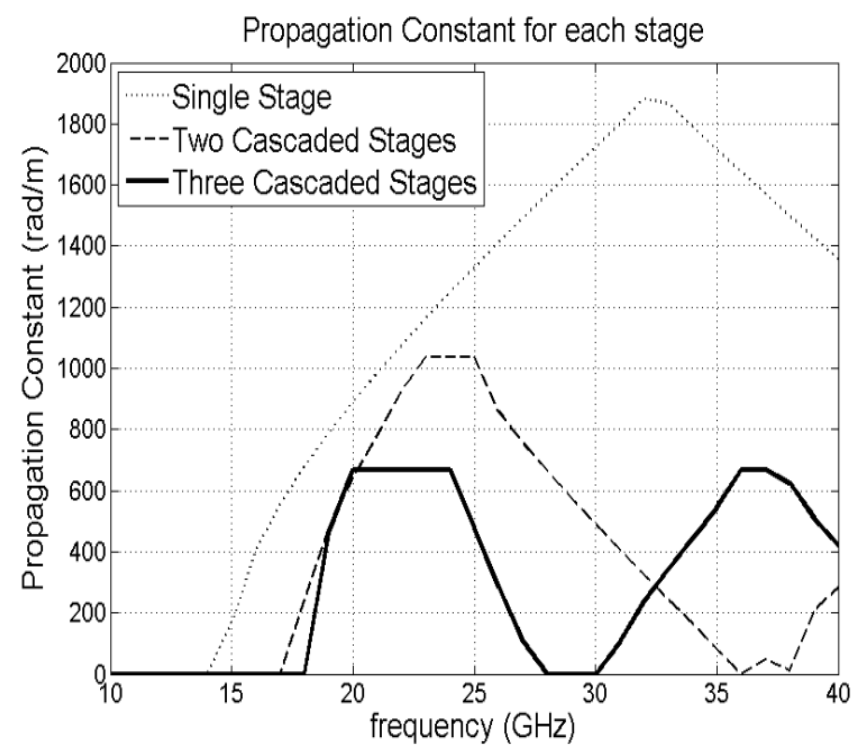

Fig. 5 Propagation Constant for each stages.

\section{E. Efficiency check :}

A fitness function defined in section 3.2 shows a matching between proposed model and rectangular waveguide model (when $\mathrm{W} 1=\mathrm{W} 2=\mathrm{W} 3=\mathrm{W}$ ),

Let, $\mathrm{Q}=\mathrm{Q}_{\mathrm{L}}$ of proposed design when $\mathrm{W}=3.97 \mathrm{~mm}$, $\mathrm{W} 1=3.07 \mathrm{~mm}, \mathrm{~W} 2=2.17 \mathrm{~mm}, \mathrm{~W} 3=1.27 \mathrm{~mm}$ and $\mathrm{Q}^{\prime}=\mathrm{Q}_{\mathrm{L}}$ of filter when $\mathrm{W}=\mathrm{W} 1=\mathrm{W} 2=\mathrm{W} 3=3.97 \mathrm{~mm}$. For single cascaded stages $Q^{\prime}=0.5432$ and $Q=0.889$ and Fitness is $61.10 \%$ by (7) and For three cascaded stages $\mathrm{Q}^{\prime}=3.17$ and $\mathrm{Q}=3.18$ and Fitness is $99.94 \%$ by (7).

\section{F.Comparison :}

A comparison is made between proposed model (two and three stage cascaded SIW Filter) and model proposed in [1], The model proposed in this paper has small transition duration,(from minimum propagation to maximum propagation) of $2 \mathrm{GHz}$

A.Table-II Comparison with Existing model

\begin{tabular}{|l|l|l|l|}
\hline $\begin{array}{l}\text { S. } \\
\text { No. }\end{array}$ & Filter & Stages & $\begin{array}{l}\text { Transition } \\
\text { duration }\end{array}$ \\
\hline 1. & In [1] & single & $18.2 \mathrm{GHz}$ \\
\hline 2. & \multirow{2}{*}{ Proposed Filter } & single & $18 \mathrm{GHz}$ \\
\cline { 3 - 4 } & & Three cascaded & $2 \mathrm{GHz}$ \\
\hline
\end{tabular}

\section{CONCLUSION}

A new approach to design Substrate Integrated Waveguide Filter has been proposed by cascading SIW stages of same length with varying Iris window and experimented for Propagation constant for different stages. It is possible to design a filter with control propagation constant by reducing its transition duration. The transition duration of propagation for the design is $2 \mathrm{GHz}$ and shows $99.94 \%$ matching with rectangular waveguide.

\section{References}

[1] Mehdi salehi, Isfandias Mehrshashi, "A Closed Formula for Dispersion Characteristics of Fundamental SIW Modes", IEEE Microwave and Wireless Components Letters, vol. 21.No.1, pp.4-6, January 2011

[2] Aman Dahiya, Anand Pratap Singh Sengar ,Dr. Dhirendra Kumar Dwivedi, Dr.Arun Kumar, "A Critical Review of Substrate Integrated Waveguide for Microwave Applications", Second International Conference on Computational Intelligence \& Communication Technology, pp.495-499, 2016

[3] Natalia Leszczynska, Mariusz Klinkosz, Michal Mrozowski, " Substrate-Integrated Waveguide (SIW) Filter Design Using Space Mapping”, 2016

[4] Weiping Li, Zongxi Tang, and Xin Cao, "Design of a SIW Bandpass Filter Using Defected Ground Structure with CSRRs",, Active and Passive Electronic Components Volume 2017, pp.1-6, Hindawi ,January 2017

[5] Xiao-ping chen, Ke Wu,"Substrate Integrated Waveguide Cross-Coupled Filter With Negative Coupling Structure", IEEE Transaction on Microwave Theory and Techniques, vol. 56,no.1, pp. 142-149,Jan 2008. 
[6] Nuria Esparza, Pablo Alcón, Luis Fernando Herrán, Fernando Las-Heras, "Substrate Integrated Waveguides Structures Using Frequency Selective Surfaces Operating in Stop-Band (SBFSS-SIW)", IEEE Microwave and Wireless Components Letters, pp. 1-3, 2016

[7] Xiao-Ping Chen, Liang Han, and $\mathrm{Ke} \mathrm{Wu,} \mathrm{"Synthesis} \mathrm{and}$ Design of Substrate Integrated Waveguide Filter Using Predistortion Technique", Proceedings of Asia-Pacific Microwave Conference, 2007.

[8] Tae-Soon Yun, Hee Nam, Ki-Byoung Kim, and Jong-Chul Lee, "Iris Waveguide Bandpass Filter Using Substrate Integrated Waveguide (SIW) for Satellite Communication”, APMC2005 Proceedings, 2005.

\section{AUTHORS PROFILE}

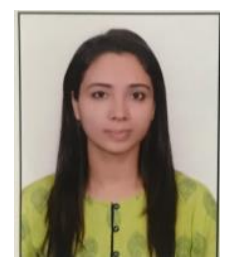

Soumya Jain, M.E. Scholar, Dept. of Electronics \&Telecommunication Engineering ,Jabalpur Engineering college, Jabalpur, India. And has done Bachelor of Engineering from Vindhya Institute of Technology and Science, Jabalpur, India.

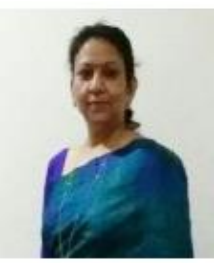

Dr. Agya Mishra working as Assistant Professor in Dept. of Electronics \& Telecommunication Engineering, Jabalpur Engineering College, Jabalpur, India. She has done her Ph.D. and M.Tech from Maulana Azad National Institute of Technology, Bhopal, India. and Bachelor of Engineering from Government Engineering College, Ujjain, India. Her specializations are in the field of Digital Signal \& Image Processing, Higher Order Neural Network, Wavelet transform applications, artificial intelligence computational Intelligence etc. 